\title{
Mobile Micro-Robots Ready to Use: Alice*
}

\author{
Gilles Caprari and Roland Siegwart \\ Autonomous Systems Lab \\ École Polytechnique Fédérale de Lausanne (EPFL) \\ CH-1015 Lausanne, Switzerland \\ \{gilles.caprari, roland.siegwart\}@epfl.ch
}

\begin{abstract}
This paper presents the latest developments around our mobile micro-robot Alice. This small robot is the starting point driving and enabling enhancements in locomotion, energy, communication, perception and control. A set of new features and new HW modules is described. The robot itself is $\sim 2 \times 2 \times 2 \mathrm{~cm}^{3}$ and is able to move, sense, receive remote commands and locally communicate with neighbor robots. Extension modules implement a long-range sensor, radio communication, a linear camera and an energy pack. The current projects in biomimetic, collective and evolutionary robotics using this set of tools are also shortly explained.
\end{abstract}

Index Terms - Mobile robotics, micro robotics, modular systems, micro-mechatronics.

\section{INTRODUCTION}

Mobile Micro-Robots (MMRs) are getting better and better because the necessary technology is progressing fast and also because research labs were recently involved not only in the design of prototypes but also in providing a set of modular solutions to easily use these robots [1][2][3]. The goal of our activity is also to extend continuously the capabilities of mobile micro-robots. It is also a need when using MMR in research projects, exhibition and application oriented demonstrations. Creating new features, enhancing the existing capabilities and building software and hardware modules easy to re-use, gives more flexibility and opens new possibilities to use MMRs in a multitude of future applications.

This paper presents enhancements in all the subsystems of a MMR, that is in robot mobility, actuation, energy management, perception, communication and control. Moreover, it describes some working tools and a couple of the most recent projects using Alice as the robotics platform.

The discussion covers the new developments over the last
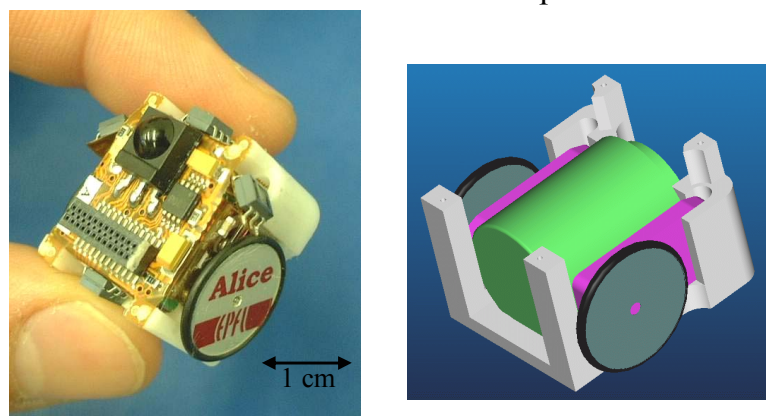

Fig. 1. Alice 2002 basic configuration. 2 watch motors, a rechargeable NiMH battery, 4 IR proximity a PIC 16F877 microntroller
3 years at the Autonomous Systems Lab related to the robot Alice 2002 (Fig.1, [4]) which was the starting system and the motivation at the same time. Not too many details are given on every new mentioned aspects but rather a quite exhaustive overview to see the great potential of this integrated mobile micro-robotics set.

\section{RoBOT MOBILITY}

Alice and most of the other MMR are restricted to very flat environments. Exploring other types of terrains and locomotion is very important but also very difficult because of the involved mechanic complexity and the need of efficient actuators. A couple of realizations in this direction are described in the following sections.

\section{A. All Terrain Alice}

All Terrain Alice (Fig. 2a) is very similar to Alice 2002. The circuit and all the components are exactly the same. Only the locomotion system was changed. The motivation for the new design was to increase the mobility of micro-robots. The system with caterpillar treads was chosen among others because it can be compact and relative simple. Another motivation is that with this solution the number of motors is not increased. However, the friction in the mechanism requires a higher torque from the motor. Taking the output of the watch motor from the hour axle instead of the minute axle, we can gain about a factor 12 for the torque. Of course the speed will be divided by 12 . This robot performs well on several terrains from carpet to sand. It can negotiate easily a slope of 40 degrees. The only problem is that the center of gravity is too high and thus the robot is not very stable in some postures.

\section{B. LamAlice}

The off-road LamAlice (Fig. 2b, [5]) is designed linking together two Alice robots. Exactly the same components as
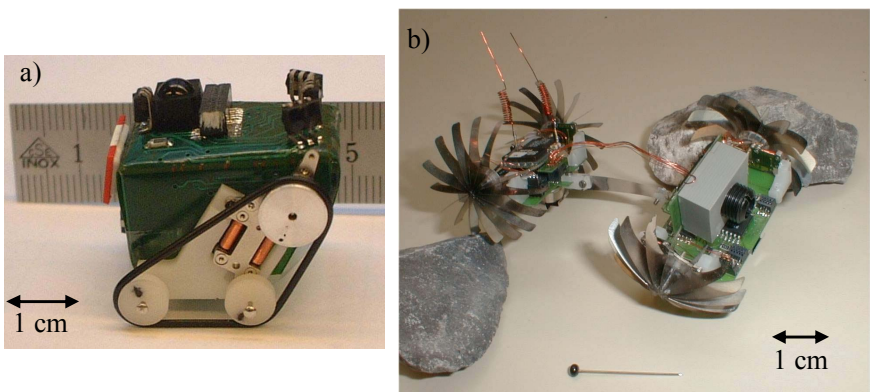

Fig. 2. Extended mobility of MMR. a) AliceAllTerrain with belts. Volume 1 inch $^{3}$. b) LamAlice with camera, radio module and wheels made of blades.

\footnotetext{
* This work is partially supported by the Swiss National Science Foundation and by the Future and Emerging Technologies program of the European Community.
} 


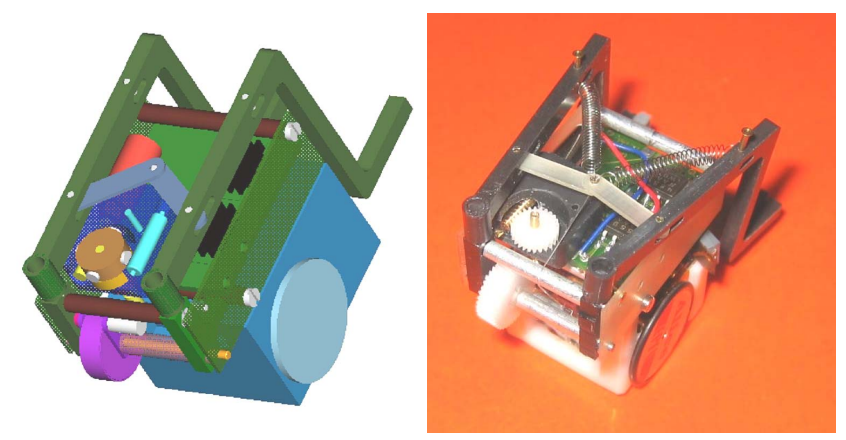

Fig. 3. Gripper module. Using only 1 small DC motor it is able to grasp and lift an object in front of Alice.

Alice are used: motors, microcontroller, sensors and connectors for the extensions. The software is similar and the same radio module can be used. An additional camera module was developed for this robot using a low power CMOS camera. Using the similar concept and the same components of Alice, this robot has extremely long power autonomy of about 20 hours. LamAlice is $11 \mathrm{~cm}$ long, $6 \mathrm{~cm}$ wide and $4 \mathrm{~cm}$ high and has a weight of 30 grams.

An improvement is made with the special wheels that are flexible and much bigger. These permit mounting on obstacles in a smooth manner. These wheels require a higher torque than the ones of Alice and thus they are mounted on the hour hand of the watch motor instead of the minute hand, as is the case for the Alice robot. Although the additional gear ratio of 12 between hour and minute axis, the speed of the robot is about $10 \mathrm{~mm} / \mathrm{s}$. This speed is more than acceptable for the envisioned application, which is planetary exploration. In fact this robot was developed for the European Space Agency (ESA) as a nano-rover demonstrator.

\section{ACTUATION}

The tasks to be performed by these robots could require different sensing and actuation capabilities. For some clustering experiments the presence in a particular position may be sufficient. Sensing and transmitting the gathered information is the next step. But to physically modify the environment the robot has to exert forces as for example pushing objects, displacing or grasping materials.

For locomotion on flat terrain or at low speed the power required is in the range of few milliwatts and thus watch motors are well suited. As we speak of mobile robots, most of the time the motors for locomotion are turned on and thus should be energetically efficient. For the movement of a gripper involved in grasping and lifting there is a shorter need of power but the peak value could be high. For this reason the gripper module (Fig. 3) is powered by a small $4 \mathrm{~mm} \mathrm{DC}$ motor delivering up to $50 \mathrm{~mW}$. The reduction is made with 2 worm gears to achieve a ratio of 500 in a small volume. With a particular mechanics, the movement of grasping and lifting are achieved with just 1 motor. In the lower position, the 2 arms are kept open by a pulling wire attached on the gears. Rising the arms, the wire get loose and 2 springs keep the arms closed while the gripper can continue to lift the grasped object. Consumption is more than $100 \mathrm{~mW}$ but the gripper is opened and lifted in a couple of seconds.

\section{ENERGY}

There are several energy sources and storage methods. The ones practicable for mobile micro-robots are batteries, capacitors, rechargeable batteries and solar cells (Fig. 4a). A very advantageous combination is a rechargeable battery with a solar panel. The latter produces energy when sufficient light is available and the battery provides a constant current even if the light is temporarily not sufficient. With this idea an extension energy module (Fig. 4b) was developed in 20032004. It fits on top of Alice 2002 and was tested in this configuration. Several solar panels can be used on this module as the input voltage is adjustable and a power management circuit controls both working input and output voltage. The core of that circuit is a DC-DC converter, which is switched by analog comparators so that the maximum power is taken from the photovoltaic cells. We tested a commercial solar

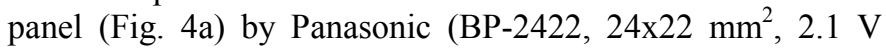
nominal, $6.6 \mathrm{~mA} \max$ ), a set of photodiodes (28 x BPW34, 4 parallel groups of 7 in series) and a high performance solar cell from Spectrolab (TASC, $2.2 \mathrm{~cm}^{2}, 2.5 \mathrm{~V}$ nominal, $31 \mathrm{~mA}$ max). The rechargeable battery is directly the one in the Alice 2002 base (Varta 3/V40H NiMH). The solution works well but too much light is incompatible with the IR proximity sensors. When there is a lot of light the solar panel generates enough energy to drive the robot but the sensors are blinded. A simple workaround is to have cycles with more light when charging and less light when the robot moves. This solution was used in a demo setup in our Lab at EPFL consisting of 3-4 robots moving in a $50 \times 80 \mathrm{~cm}^{2}$ arena. The setup is running non-stop: during the day the Alices move around, whereas in the night a strong light (4 Halogen Spot $10^{\circ} 50 \mathrm{~W}$ ) is switched on by a timer and the robots autonomously place them underneath to get recharged.

Other ways to increase the power autonomy are to save as much as possible the consumption or to add an additional battery. Alice uses very low power motors and in most cases those can even be driven with a PWM duty cycle of $80 \%$. The active proximity sensors are refreshed at a reasonable rate of $20 \mathrm{~Hz}$ and the standard frequency of the CPU is as low as $4 \mathrm{MHz}$. With these solutions the Alice can run up to 10 hours out of a $40 \mathrm{mAh}, 3.6 \mathrm{~V}$, NiMH battery.
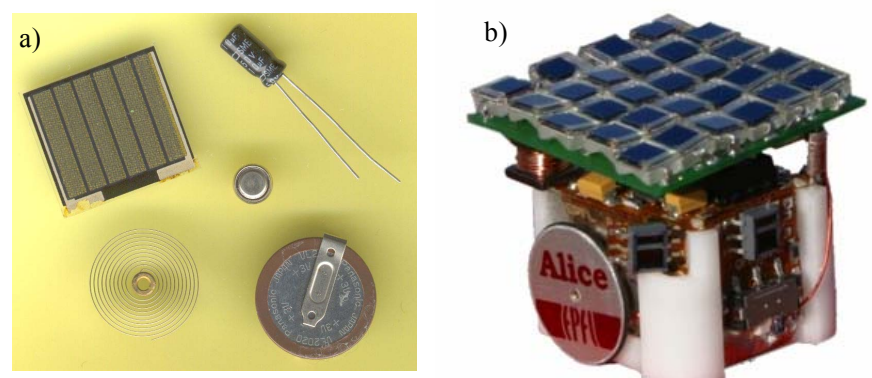

Fig. 4. Energy solutions. a) Photovoltaic cell, super cap, watch coin battery, spring, Lithium backup battery. b) Alice with the solar module powered by 28 BPW34 photodiodes. 

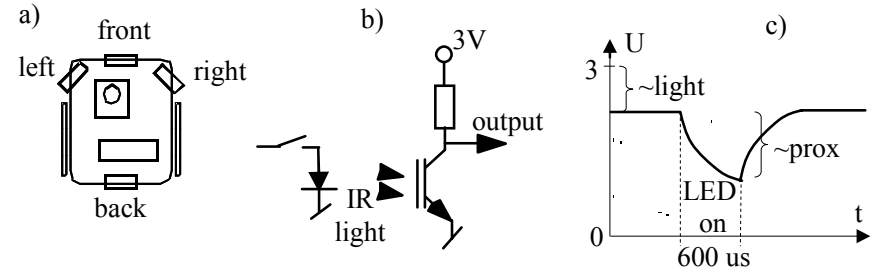

Fig. 5. IR proximity and light sensors. Placement on the robot, circuit and timing of the output signal.

For particular applications where extreme long power autonomy is required, an extension power module is available. This includes a lithium-polymer (LiPo) rechargeable pack with $190 \mathrm{mAh}$ in addition to the $40 \mathrm{mAh}$ of the on-board $\mathrm{NiMH}$ cell. It is used for example in the extension for the "ANT evolution" project where the robot evolves and is tested over a long period (see Fig. 7 and section VIII B).

\section{PERCEPTION}

Perception is a key issue for the future use of mobile robots. From the physical sensor, to the electronics, up to the interpreting algorithm, useful information has to be extracted from the sensor signal in order to understand the environment and take the correct decisions.

\section{A. Proximity and Light Sensors}

The IR proximity sensors are the basic sensors for Alice as for most other miniature mobile robots. These are simple to implement, include a sensing IR photodiode and an emitting IR diode, allowing ambient light and proximity measure. The output signal diagram in Fig. 5c shows the method used. To get the ambient light information, the photodiode current is read without turning on the IR LED. For the proximity, the Led is turned on during 600 us before reading the new response on the photodiode. The difference between this value and the ambient light is proportional to the proximity of an object placed in front of the sensor, and this more or less insensitive to the ambient light. The Alice base has 4 SFH9201 sensors mainly facing forward because it is the preferred direction of movement. For applications requiring a more uniform circular perception, we added 2 sensors back left and back right.

\section{B. Long Distance Sensor}

The IR proximity sensors on the Alice base can sense objects up to 3-4 $\mathrm{cm}$ far away because of their sensibility and the limited emitted power of the IR beam supporting the measure. In order to detect objects a bit further away, a special module was designed. It uses exactly the same concept of sending an IR beam and measuring the difference with and without emission but pulses the emitter with a higher current. A capacitor is charged and discharged through 2 transistors to burst enough current ( $40 \mathrm{~mA})$ without pulling too much from the battery that would otherwise induce noise on the power supply. With this solution the maximal sensible distance is about the double, that is 6 to $8 \mathrm{~cm}$ in front of a white wall.
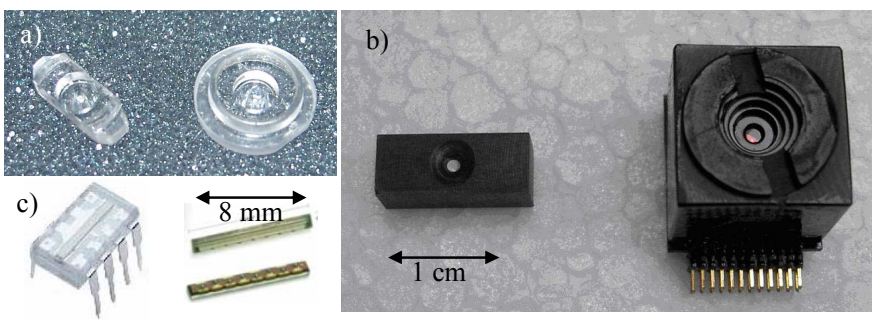

Fig. 6. Linear camera parts. a) EL20 lens cut and original. b) compact optic box and linear camera module with standard optics EL20. c) TSL3301 and the compact TSL1401CS.

\section{Linear Camera}

The robot can be equipped with a linear camera module to be able to perceive features far away. We used the sensor TSL3301 by TAOS and we created the optics holding box around it. The lens can be screwed in a standard M12x0.5 thread, typically we used an EL20 from Applied Image Group and we removed the external useless plastic. This results in a module to be plugged on top of Alice (Fig. 6b), a little bit smaller than the robot itself, and can be read out directly by the robot's main processor (PIC) with a serial protocol. The chip has $102 \times 1$ pixels and an integrated 8 bit ADC so that the PIC can easily manage the information flow. It still works at 3 Volts, and with a picture refresh rate of $50 \mathrm{~Hz}$, the consumption is $2.2 \mathrm{~mA}$. The typical features such a sensor can detect are bright spots, vertical black and white lines and their width.

The newest development regarding linear cameras is a further integration using the very compact sensor TSL1401CS from TAOS (Fig. 6c), which consists practically only by sensing surface: $\sim 8 \times 1 \times 1 \mathrm{~mm}^{3}$. Along with an optimized holding box and an in-house modified lens (Fig. 6a), the whole takes only $12.5 \times 5 \times 6 \mathrm{~mm}^{3}$, allowing an easier integration for example in the "ANT extension" module presented next.

\section{ANT Extension}

The robot requirements for the "ANT evolution" projects (section VIII. B.) are so many that a newer extension module had to be developed. The robot needs long power autonomy, has to discriminate small and big objects, white and black walls and has to communicate locally while pushing items. The solution is to integrate all the sensors and an additional battery in a compact module to be plugged on top of the robot (Fig. 7). Starting from the bottom of this extension, 2 additional proximity sensors extend the perception and the local communication area also to the rear. The big LiPo battery find place between 2 PCBs, one housing the long range IR sensor circuit and the other a voltage regulator and a switch to deliver the correct working voltage to the rest of the robot. At the higher level the miniaturized linear camera with optics gives the possibility to see white and black walls from far away. On the top, the extension connector of Alice is replicated allowing further extension and the access to the programming pins.

The module is assembled by soldering the PCBs perpendicularly, insuring at the same time mechanical and 

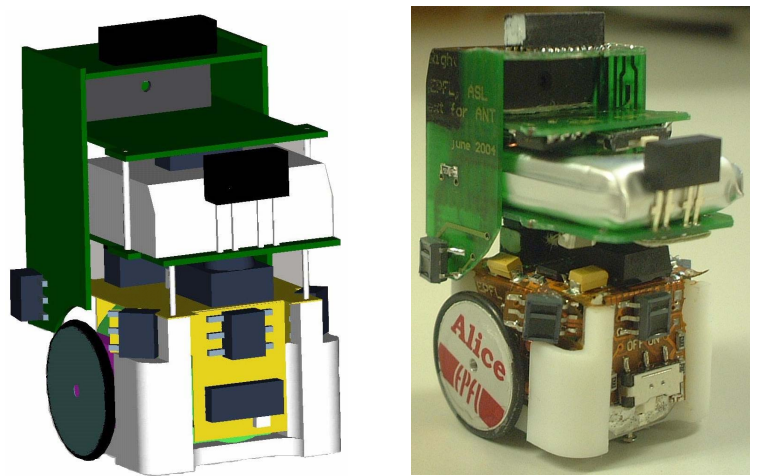

Fig. 7. ANT extension plugged on the robot Alice. It includes 2 proximity and 1 long distance sensors, a LiPo battery and a 128 pixels linear camera.

electrical connection. The overall dimensions of this powerful module are a bit bigger that the robot itself but it fulfills all the requirements and 20 units have been built and are in use.

\section{COMMUNICATION}

We can distinguish between local, short range and global, long distance communication. The local one is implemented on Alice with IR signals and is used to exchange information among nearby agents and exploiting it directly for decision and control. The long range one is supported by radio waves and is needed to receive commands, to retransmit sensor data or for supervision. The 2 following sections discuss some details, protocols and results of these 2 methods.

\section{A. Local IR Communication}

The infrared proximity sensors for environment perception (Fig. 5) can also be used for communication with other nearby robots. The communication is quite limited however it is bidirectional, works up to $4 \mathrm{~cm}$ and allows figuring out the relative geometrical configuration of the 2 communicating robots. The IR sensors are active and fully controlled by the software in the on board microcontroller PIC16F877. The protocol is coded in assembler and it is part of the timer interrupt routine, which is called every 200 us. The communication is transparent for high level $\mathrm{C}$ algorithm, which has just to put the output message in a variable (CommState), and as soon as another robot send something, a flag $($ Received ()$)$ is raised up and the incoming message is ready in another variable ( $v a l)$. The simple message is just a nibble ( 4 bits), thus values between 0 and 15 can be

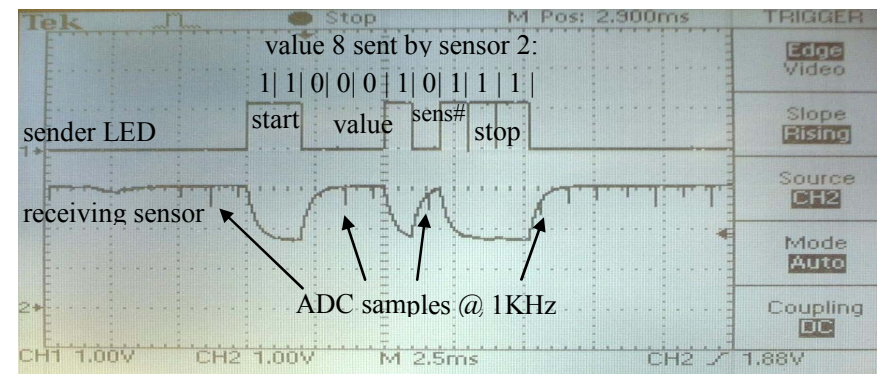

Fig. 8.Local communication. Signal on the sender and on the receiver Robot. The spikes on the received signal are do to AD sampling. The bits of the protocol are: 2 start, 4 value, 2 sensor\#, 2 stop.

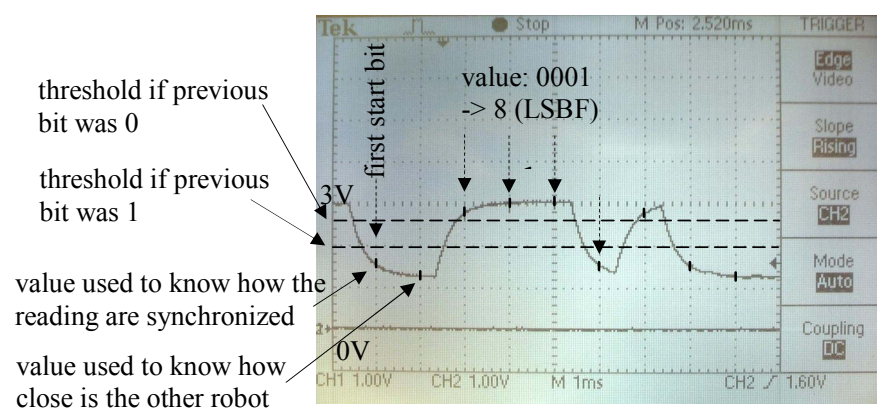

Fig. 9. Synchronization and thresholds on sensed signal. As the signal can be sampled only once per bit and the response is slow, special solution are implemented using different conditions and thresholds.

exchanged between the robots. The meaning of a specific value depends on the user software but typically it may be the robot identifier, the level of an attractive or repulsive force. If the Alices are close enough and at least a couple of sensors are facing each other, then the exchange of the messages can takes place every $50 \mathrm{~ms}$. The message includes the 4 information bits and the identifier ( 2 bits) of the transmitting sensor. The receiving robot receives the message with only 1 sensor and thus the user software knows which sensor (she) was used by the other robot and on which sensor (me) the communication was received. This permits understanding the relative position of the talking Alices.

The protocol has to establish which robot is the communication master and which one is the slave. It has to detect the start of the message, read the bits and check if it really was a message. For these reasons the signal (Fig. 8) has 2 start bits and 2 stop bits. Note that because of the limited computing power the message can be sampled only one time per bit and the position of the sample among the bit is of course not known. Moreover the sensor is quite slow and thus reconstructing the message is not easy and can induce some errors. The user software has to take this into account handling this communication feature as something not $100 \%$ secure but only more or less $90 \%$ precise. A couple of methods (Fig. 9) are used in order to decrease the communication errors:

- For a good synchronization the level of the second start bit has to be lower than the first.

- These 2 values are used to know how the readings are synchronized and how to adapt the thresholds.

- To cope with the slow sensor response, the thresholds are different depending if the previous received bit was logical 1 or logical 0 .

The results of the tests done with the robots give a quality around $90 \%$, speed about 10 messages per second, consumption of $1.5 \mathrm{~mW}$ when only listening and $3 \mathrm{~mW}$ at high communication rate.

\section{B. Radio Communication}

The robot is able to communicate via a radio link with other robots or with a host computer. This feature is of high interest to supervise the status of a robot by means of a host computer or to exchange information between robots even if they do not see each other. 


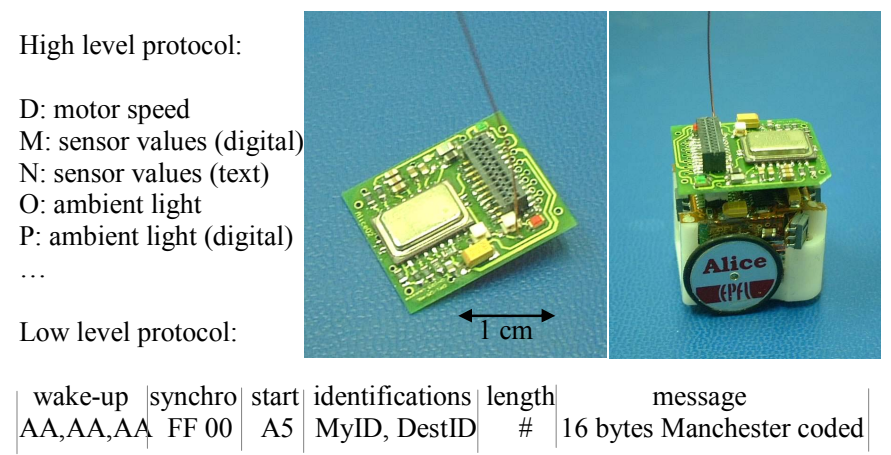

Fig. 10. Recent radio module using the TR1001 chip and the relative low level and high level communication protocols.

The first developed radio module contained a HX1000 device as transmitter and a RX1020 as receiver, both working at $433.92 \mathrm{MHz}$ and made by RF Monolithics inc (www.rfm.com). The transmission of data in both directions was tested up to a distance of about 10 meters at $1 \mathrm{Kbit} / \mathrm{s}$.

The actual radio module (Fig. 10) uses a newer generation transceiver chip from RFM, the TR1001 working at $868 \mathrm{MHz}$, $115 \mathrm{Kbit} / \mathrm{s}$. The consumption is quite low: $\mathrm{RX} \sim 6 \mathrm{~mW}, \mathrm{TX} \sim$ $24 \mathrm{~mW}$. The chip is directly controlled by the Alice microcontroller with the RS232 port at $125^{\prime} 000$ baud and 2 digital control signals. The serial line is constantly monitored for incoming messages respecting the correct protocol. This task is quite CPU time consuming and the quartz frequency of the microcontroller had to be doubled to $8 \mathrm{MHz}$. Also for this reason the protocol was kept relatively simple. This is composed by a waking-up sequence followed by 2 synchronization bytes, a start byte, the owner and destination identifiers (each 1 byte), the message length and finally the message itself. For a correct functioning of the radio chip, the digital sequence has to be DC balanced and thus a byte-wise Manchester encoding was implemented. This is easily done and permits detecting errors as well. If everything is correct the addressed robot sends back an acknowledgment to the requesting station. A request message is started by a capital letter and the relative acknowledgment is simply the corresponding lowercase character. Broadcast is possible setting the destination address to 255 . With the overhead of the protocol the real useful bandwidth is about $30 \mathrm{kbit} / \mathrm{s}$. Some typical messages are: motor speed commands, proximity sensors or ambient light measures.

The newest tendency is to use chips working in the 2.4 $\mathrm{GHz}$ band and doing frequency hopping. Bluetooth is a nice standard but there are other chips not following that standard, which allow more freedom and potentially lower power consumption. A newer radio module using such a component (nRF2401 from Nordic VLSI) is under development and will be soon operational.

\section{CONTROL}

There are different ways to control robots, ranging from totally remote controlled to completely autonomous. Several overlapping feedback loops can coexist, be added or taken out.

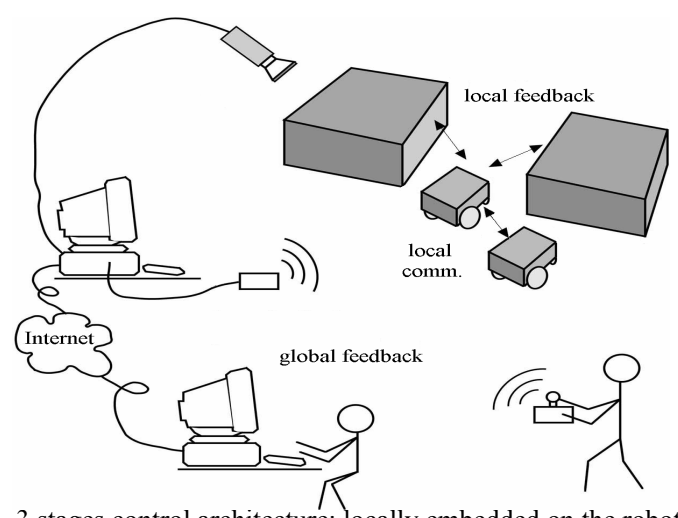

Fig. 11. 3 stages control architecture: locally embedded on the robot, nearby host computer and human operator.

As a general framework we propose the 3 stages control and for the low level robot loop we summarize the newly implemented Neuronal Network embedded on the Alice OS.

\section{A. 3 Stages Control}

The control method proposed is somewhere between autonomous and remote controlled. The robot has some preprogrammed basic behaviors and communication abilities. Using this base the control architecture puts together an intelligent general behavior depending on the task. The underlying software architecture with the robot operating system permits the pseudo-parallel execution of all the subtasks. This is the lower level control loop. To add intelligence to the system, an external host PC can help the robot. It might for example act seeing and analyzing the scene with an overhead camera. The visual feedback closes the second control stage. The last and upper control loop is made by a human operator specifying the global goals or with manual intervention if everything else fails.

\section{B. Embedded Neuronal Network}

To test bio-inspired evolutionary control approaches, a structure of a neuronal network (NN) is programmed in the Alice microcontroller. This is used for the "ANT evolution" project (see section VIII. B.) where the NN controls the behavior of the robot. The sensor values are fed as input in the $\mathrm{NN}$ and the calculated outputs set the motor speeds. The onboard microcontroller (PIC16LF877) has an 8-bit CPU, no hardware multiplication, no floating points and limited memory. This imposes severe constraints to the implementation of such a controller and forces to use limited resolution, and several tricks like shifting instead of division, tables instead of exponential functions and a careful handling of the significant value of the 8 bit variables.

In order to calculate the value of a neuron from the incoming inputs of the other neurons the following calculation is performed. The neuron membrane potentials and the synaptic weights should be interpreted as float in the range $(-1 ; 1)$ and $(-2 ; 2)$ respectively but are both represented and stored as 8 bit integer in the range [-127;127]. The 8 by 8 bit multiplications are done software-wise and the 16 bit results from all the synapses are summed up in a 32 bit temporary variable. This is shifted by a dividing factor and limited in 
order to get again a value fitting in 8 bits. Finally the actuation function is applied in the form of a table representing the function $\tanh ()$ giving as a result again an 8-bit variable. This represents the calculated membrane potential of the goal neuron. The dividing factor depends on the interpretation of the 8 bit variables and from the active region of the $\tanh ()$ table (typically from -4 to 4 ). In our case, with the mentioned scaling of the variables and the table, the dividing factor is realized by a simple right shift of 8 bits.

The tested NN is composed by 9 inputs ( 5 proximity, 2 pixels, 1 local comm. input and 1 bias), 4 internal +1 bias and 3 outputs ( 2 motors, 1 local comm. output). The $\mathrm{NN}$ is fully connected, but there are no direct input-output and no feedback links, resulting in 51 synapses. The quartz frequency of Alice was increased to $10 \mathrm{MHz}$ giving an update calculation time of less than $5 \mathrm{~ms}$ while running in parallel all the other tasks like sensor sampling, motor control and communication. The $\mathrm{NN}$ program is written in $\mathrm{C}$ and together with all the rest of the Alice control code, it uses about $3 \mathrm{~K}$ of program memory and 150 bytes of RAM. This demonstrates as well that small microcontrollers have sufficient power for mobile micro-robots.

\section{PROJECTS AND APPLICATIONS}

Some on-going projects are shortly mentioned in order to show the kind of usages that can be expected from a MMR. This paper would like to give an overview of the actual capabilities of our micro-robotics system and for a deeper description please refer to the project publications.

\section{A. European Project LEURRE}

This projects aims at understanding and controlling mixed societies of animals and robots. Alice was used as first approach of MMR to be put together with insects (cockroaches) and to test the implementation of embedded insect-like behaviors [6][7].

\section{B. “ANT evolution” Project}

Alice is experimentally used to study the "Evolution of Cooperation and Labor Division in Artificial Ants" [8][9]. Inspired by the cooperation in real ants, the goal is to understand these aspects using computer simulation and real robots as proof and demonstrators. Three labs participate to the project: prof. Keller's group at Uni Lausanne; LIS by prof. Floreano and ASL1 by prof. Siegwart, both at EPFL. In the experiment, a group of Alices has to collect food item and push them toward the nest. Small objects can be pushed by a single robot but bigger objects require the cooperation of at least 2 robots and give more reward. The nest is seen with the linear camera; the food items with the proximity sensors and the local communication enable active cooperation among teammates. The behavior is controlled by a NN where the weights are artificially evolved with runs mostly in simulation and partly in the real setup. The dynamics of the evolution will suggest the best conditions, under which cooperation takes place.

\section{Exhibition in Museum}

Since end 2004, 5 Alices will be shown 1 year long at the museum Fondation Claude Verdan, musée de la main (www.verdan.ch). Simple behaviors implemented in the robot like stop and go, attraction to a light held by the visitor, obstacle avoidance and a blinking LED, permit interacting with the people and eventually induce reflections on emotions and artificial intelligence.

\section{CONCLUSION}

During the last years there were many improvements on the extensions of Alice, which proves that the robot itself is a good basis but there is a general need for more sensing and communication capabilities. Some effort in mechanical design has to be done as well in order to increase the mobility of MMRs. In the nature there is plenty of inspiration and examples to compete with. Those set the benchmarks very high and leave us a long way to cover. However with the work done so far, once more it was shown that mobile micro-robots can accomplish various tasks with an increasing complexity.

\section{ACKNOWLEDGMENT}

The LEURRE project is funded by the European Community (IST-FET). The Swiss participation as well as the ANT project are supported by the Swiss National Science Foundation. Thanks to Alexandre Colot, Jean-Christophe Zufferey and Markus Waibel for their comments and hints; André Guignard for his valuable technical help and all the students that contributed to some developments: Matthias Greuter, Walter Karlen, Vasco Medici, Marc Freese, Sveva Ambrosetti, Amy Lafrance, Davin Sufer and Linus Gasser.

\section{REFERENCES}

[1] T. Fukuda, H. Mizoguchi, K. Sekiyama, F. Arai, "Group Behavior Control for MARS (Micro Autonomous Robotic System)", Proc. of Int'l Conf. on Robotics and Automation, ICRA '99, pp. 1550-5, 1999.

[2] F. Mondada, E. Franzi, P. Ienne, "Mobile robot miniaturization: A tool for investigation in control algorithms", Proc. of the $3^{\text {rd }}$ Int. Sym. On Experimental Robotics, pp. 501-513, 1993.

[3] Navarro-Serment, L.E., Grabowski, R., Paredis, C.J.J., and Khosla, P.K. "Millibots: The Development of a Framework and Algorithms for a Distributed Heterogeneous Robot Team," in IEEE Robotics and Automation Magazine, Vol. 9, No. 4, 2002.

[4] G. Caprari, T. Estier, R. Siegwart, "Fascination of down scaling: Alice the sugar cube robot", J. of Micromechatronics, vol. 1, no. 3, pp. 177-89, 2002

[5] M. Lauria, et al, "LameAlice, a nanorover for planetary exploration", Video Proceedings of ICRA'03, Teipei, Taiwan, 2003.

[6] G. Caprari, A. Colot, R. Siegwart, J. Halloy, J.-L. Deneubourg, "Building Mixed Societies of Animals and Robots", Robotics \& Automation Magazine. In press.

[7] LEURRE web site, "Artificial life control in mixed societies", http://leurre.ulb.ac.be

[8] ANT web site, "Evolution of Cooperation and Labor Division in Artificial Ants", http://asl.epfl.ch/research/projects/EvoAnts/

[9] A. Perez-Uribe, D. Floreano, L.Keller, "Effects of group composition and level of selection in the evolution of cooperation in artificial ants", $7^{\text {th }} E U$ Conf. on Artificial Life, ECAL'2003, Springer, pp. 128-137, 2003. 